\title{
IS THE ATOM THE ULTIMATE MAGNETIC PARTICLE?
}

\author{
By Arthur H. Compton AND Oswald Rognley.
}

SYNOPSIS.

Effect of Magnetization of Crystal on Intensity of X-ray Reflection; Theory.-The intensity of reflection of X-rays from a crystal depends upon the arrangement of the atoms within the crystal and upon the arrangement of the electrons within the atom. If the ultimate magnetic particle is a group of atoms, such as the chemical molecule, magnetization of the crystal will change the orientation of the group and hence change the positions of the individual atoms. If the ultimate magnetic particle is the atom, magnetization will change the orientation of the atom and hence alter the arrangement of the electrons. In either case magnetization of a crystal should be accompanied by a change in the intensity of a beam of X-rays reflected from its surface.

Effect of Magnetization of Crystal on Intensity of X-ray Reflection; Experiment.Such an effect was sought for by reflecting $X$-rays from a crystal of magnetite and measuring the intensity of the reflected beam by a sensitive balance method. The test was made on the first four orders of reflection from the natural (III) face when the crystal was magnetized perpendicular to its reflecting surface and on the third order when magnetized parallel with this surface. On magnetizing the crystal to $I / 3$ of saturation and on demagnetization, no change in intensity of the reflected beam was observed, though a variation of I per cent. would have been detected.

Molecule as Ultimate Magnetic Particle.-A displacement of the atoms due to magnetization by $\mathrm{I} / 3$ ooth of their distance apart would have produced a detectable effect. This experiment therefore affords a strong confirmation of the conclusion reached by $\mathrm{K}$. T. Compton and E. A. Trousdale that the elementary magnet is not a group of atoms.

Motion of Elementary Magnet in Strong Magnetic Field.-An argument is presented which shows that when saturation occurs the elementary magnets very probably have their axes nearly parallel with the direction of magnetization.

Atom as Ultimate Magnetic Particle.-Subject to the validity of this conclusion, it is not found possible to explain the negative result of the experiment if the atom as whole acts as the elementary magnet. Certain other explanations are also discussed and found unsatisfactory.

Electron or Positive Nucleus as Ultimate Magnetic Particle.-Either of these conceptions is in accord with the result of the experiment, but auxiliary evidence favors the electron as the probable elementary magnet.

A FEW years ago K. T. Compton and E. A. Trousdale described in this journal ${ }^{1}$ an experiment which led them to the conclusion that the ultimate magnetic particle is not any group of atoms, such as the chemical molecule, but is rather the atom or something within the atom. It has long been a favorite method of explaining the magnetic

${ }^{1}$ K. T. Compton and E. A. Trousdale, Phys. Rev., 5, 3I5 (I9I5). 
properties of matter to interpret them in terms of the magnetic moment of electrons rotating in orbits. This hypothesis has seemed to receive support by the successes of the Rutherford-Bohr theory of atomic structure, which assumes the atom to consist of electrons revolving in orbits in such a manner that the atom must possess a large magnetic moment. Furthermore, the experiments of Barnett, ${ }^{1}$ Einstein and de $\mathrm{Haas}^{2}$ and J. Q. Stewart ${ }^{3}$ have shown that the magnetization of iron is unquestionably accompanied by a change in angular momentum of the order of magnitude to be expected if the iron's magnetic properties are due to electrons revolving in orbits.

Experimental.-We have accordingly performed an experiment designed to test the hypothesis that the elementary magnet in a ferromagnetic substance is an atom consisting of rotating rings of electrons. In this experiment an attempt was made to detect a difference in the intensity of a beam of X-rays reflected from a crystal of magnetite when the crystal was magnetized and when unmagnetized. ${ }^{4}$ It will be assumed for the present that all the elementary magnets of which a ferromagnetic substance is composed are arranged with their axes parallel with the magnetic field when the substance is magnetically saturated. If these ultimate magnetic particles are the individual atoms, the orientation of the atoms due to magnetization of the crystal will change the positions of the electrons of which the atoms are composed. In virtue of the fact that the intensity of a beam of $\mathrm{X}$-rays reflected from a crystal face depends upon the arrangement of the electrons in the atoms which make up the crystal, such a shift of the electrons should make itself known by changing the intensity of the reflected beam.

Consider for example a crystal composed of atoms of the Rutherford type, each atom having all its electrons arranged in the same plane and perpendicular to its magnetic axis. When the crystal is unmagnetized, the axes of the electronic orbits will be oriented in all possible directions, so that most of the electrons will be at an appreciable distance from the mid-planes of their atomic layers. If, however, the crystal is magnetically saturated perpendicular to the reflecting face, the electronic orbits will all lie parallel to this face. The electrons will now, therefore, be in the midplanes of the layers of atoms which are effective in producing the reflected beam. Such a shift of the electrons should produce a very considerable increase in the intensity of the reflected beam of

1 S. J. Barnett, Phys. Rev., 6, 240 (I9I5).

2 Einstein and de Haas, Verh. d. deutsch. Phys. Ges., I7, r52 (I9I5).

3 J. Q. Stewart, Phys. Rev., II, Ioo (I9r8).

${ }^{4}$ Cf. A. H. Compton and Oswald Rognley, Science, 46, 4I5 (I9I7); and Phys. REv., II, I32 (I9I8) for preliminary accounts of this work. 
$\mathrm{X}$-rays, since the rays scattered by all the electrons will now be in the same phase. If the crystal is magnetized parallel to the reflecting face, the turning of the orbits will carry the electrons farther on the average from the middle of their atomic layers, the phase difference between the rays scattered by the different electrons will be greater, and a decrease in the intensity of reflection should result. If, on the other hand, the electrons are arranged isotropically in the atom, or if the atom is not rotated by a magnetic field, which would be the case if it is the individual electron or the positive nucleus that is the ultimate magnetic particle, no such change in the intensity of the reflected beam should be observed.

In our search for this effect a null method was employed. The ionization due to the beam of X-rays reflected from a crystal of magnetite was balanced against that due to a beam of the same wave-length reflected from a crystal of rock-salt, so that a very small change in the relative intensity of either beam could be detected while variations in the X-ray tube itself had but little effect. The experimental arrangement is shown diagrammatically in Fig. I. From the target $A \mathrm{X}$-rays fell upon

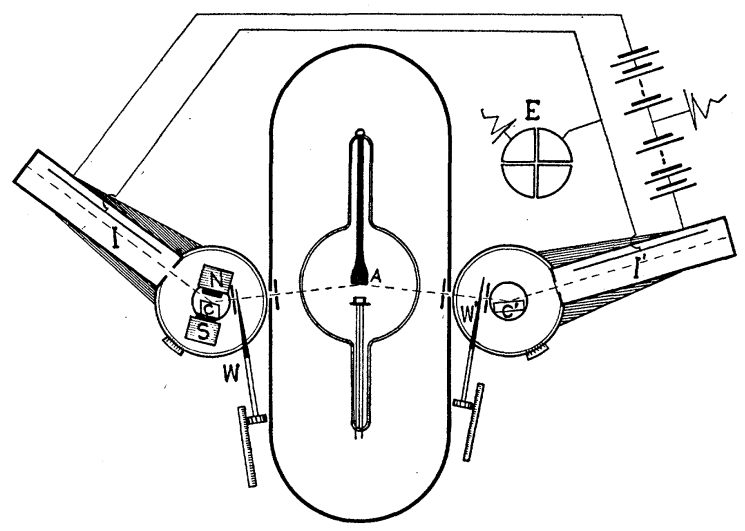

Fig. 1.

the crystals $C$ and $C^{\prime}$, and were reflected into the ionization chambers $I$ and $I^{\prime}$. One of these chambers was at a positive and the other at a negative potential, and their electrodes were both connected to the same pair of electrometer quadrants. Thus if the ionization current was the same in both chambers there was no deflection of the electrometer. The beams could be balanced accurately against each other by use of the aluminium absorption wedges $W$ and $W^{\prime}$, operated by means of micrometer screws. The crystal $C$ was cemented to one pole of an electromagnet with a laminated core, so that by changing from direct to alter- 
nating current which could be gradually reduced to zero it was possible to magnetize or demagnetize the crystal at will.

For the source of X-rays we used a Coolidge tube with a molybdenum anticathode, kindly supplied by Dr. W. D. Coolidge. As the source of high potential, a Snook-Roentgen machine was employed. The first order of the molybdenum $\alpha$ line $(\lambda=.72 \mathrm{I}$ A. U.) was always reflected into the ionization chamber $I^{\prime}$, while different orders of the same line were reflected by the magnetite crystal into the chamber $I$. It was possible with this arrangement to detect with certainty variations in the relative intensity of either reflected beam as small as I per cent. Unfortunately our magnet was not strong enough to saturate the magnetite crystal. The intensity of magnetization of the magnetite was about 150 , which is just over $\mathrm{I} / 3$ of the saturation intensity for magnetite as determined by du Bois. ${ }^{1}$

The effect of magnetization perpendicular to the plane of the crystal face was investigated in the first four orders. On account of mechanical difficulties, the test was made in only the third order spectrum when the crystal was magnetized parallel to the reflecting surface. In no case was a change as great as I per cent. observed in the intensity of the reflected beam when the crystal was magnetized or demagnetized. Our experimental results therefore may be summarized by the statement that the intensity of the X-ray beam reflected from magnetite is not altered by as much as I per cent. in the first four orders when the crystal is magnetized to $\mathrm{I} / 3$ of saturation perpendicular to the reflecting surface, nor in the third order by similarly magnetizing the crystal parallel to the reflecting face.

The Orientation of the Elementary Magnets. -The conclusions which are to be drawn from this experiment depend upon the conception that we have of what occurs to the ultimate magnetic particles when a ferromagnetic substance is magnetized. It is usually supposed that these elementary magnets have their axes so oriented by the external magnetic field that at saturation they are very nearly parallel with this field. Indeed, the manner in which this conception accounts for the phenomenon of saturation was originally perhaps the strongest argument in favor of the so-called "molecular" theory of magnetism. It should be noted, however, that the phenomenon of saturation is not in itself sufficient evidence that the ultimate magnetic particles have their axes oriented along the magnetic lines of force. This phenomenon would also occur if the elementary magnets were turned through only a small angle, but from one position of stable equilibrium to another beyond which it might be incapable of turning.

${ }^{1}$ Du Bois, Phil. Mag., 29, 293 (I890). 
Perhaps the most convincing argument in favor of a complete rotation of the elementary magnets by a strong magnetic field is that which was brought forward originally by J. Swinburne ${ }^{1}$ in connection with Ewing's suggestion that the orientation of the "molecular magnets" is determined by magnetic rather than by frictional forces. Swinburne points out that on this hypothesis, if the magnetization of a piece of iron is reversed by a strong rotating field instead of by a field alternating through zero, the loss in energy should be little or nothing, for if the molecules rotate with the field no unstable movements are possible. Experiments by F. G. Baily ${ }^{2}$ on iron, by R. Beattie ${ }^{3}$ on nickel and cobalt, and especially by Weiss on pyrrhotite, ${ }^{4}$ show that this is actually the case. For large fields, where saturation is approached, the hysteresis loss per cycle when a rotating magnetic field is applied is found in every case to be only a small fraction of the hysteresis loss per cycle due to an alternating magnetic field. There would seem to be no reason for this great reduction in the hysteresis if the axes of the elementary magnets are unable to follow closely the rotating field. If seems difficult, therefore, to avoid the conclusion that the elementary magnet, whatever it may be, is free to be oriented in any direction. ${ }^{5}$ This conclusion will accordingly form the basis of our interpretation of the experiment just described.

\footnotetext{
${ }^{1}$ Cf. Encyclopædia Britannica, IIth ed., Vol. I7, p. 350.
}

The referee to whom the editors submitted this paper has offered the following comment: "It would seem that additional strong evidence is given by the experiments of Weiss and Kamerlingh Onnes in 'Researches sur L'Aimantation aux Tres Basses Temperatures,' Journal de Physique, vol. 9, pp. 555-584, roro. In this research it is shown that the moment per c. c. resulting from complete orientation of the magnets differs by only 5 per cent in the case of nickel, 2 per cent in the case of iron, and 6 per cent in the case of magnetite from the experimentally measured saturation moment at ordinary temperatures."

It is indeed difficult to explain the very nearly complete saturation which this result indicates as due to anything other than an almost perfect alignment of the elementary magnets with the external field.

2 Ibid.

3 Ibid.

${ }^{4}$ C.f. E. H. Williams, The Electron Theory of Magnetism, p. 40.

${ }^{5}$ In any case the axis of the ultimate magnetic particle must describe a closed conical surface under the action of a rotating magnetic field in order to account for the reduced hysteresis. This conical surface is the locus of the possible stable orientations of the axis. If the elementary magnet is free to align itself with the external rotating field, the apex angle of this cone will be $\pi$; but if it is free to turn through only a small angle, the conical surface will have a sharp apex. The possibility suggests itself that the axis of this cone may represent some fixed axis in the elementary magnet, such as for example an electric axis, which may be slightly inclined to the magnetic axis and about which the magnetic axis may be free to turn. But any such rotation of one axis fixed in the particle about another means a rotation of the particle as a whole about the second axis. We cannot avoid in this manner, therefore, the conclusion that the elementary magnet is rotated by a rotating magnetic field.

In nature it is usual for an object to be capable of stable orientation in one clirection, 
The Molecule as the Ultimate Magnetic Particle.-It is obvious that if the elementary magnet consists of some group of atoms within the ferromagnetic material, the arrangement of the atoms in a crystal will be greatly altered when the groups of atoms are rotated by a magnetic field. One would therefore expect, as was pointed out by K. T. Compton and E. A. Trousdale, that magnetization would change the positions of the spots in Laue photographs taken through magnetic crystals, since these positions are determined by the arrangement of the atoms. As we have seen, the negative result obtained by these experimenters indicates that the arrangement of the atoms is not greatly changed by magnetization of ferromagnetic crystals.

The experiment which we have performed gives still more definite information with regard to the displacement of the atoms in a magnetic field. It has been shown by W. H. Bragg ${ }^{1}$ that the minute changes in the positions of the atoms due to a relatively small rise in temperature is sufficient to affect appreciably the intensity of a reflected beam of $\mathrm{X}$-rays. The negative result of our experiment shows clearly, therefore, that the atoms in a crystal are not moved as far by magnetization as they are by a change of temperature well within the melting point of the crystal. In fact direct calculation shows that a displacement of the atoms by $1 / 300$ th of the distance between the successive atomic layers would have caused a detectable change in the intensity of the 4 th order spectrum. Our experiment therefore affords a very sensitive confirmation of the conclusion reached by K. T. Compton and E. A. Trousdale that the ultimate magnetic particle is not a group of atoms, such as the chemical molecule, but is the individual atom or something within the atom.

The Atom as the Ultimate Magnetic Particle.-If the atom as a whole is the ultimate magnetic particle, it is natural to assume that its magnetic moment is due to the presence of electrons revolving in orbits. The simplest example of this type is the Rutherford form of atom, in which all the electronic orbits lie in the same plane, perpendicular to the magnetic axis. Let us therefore calculate the order of magnitude of the effect due to magnetization on the intensity of a reflected beam of X-rays if the reflecting crystal is composed of atoms of this type.

Professor W. H. Bragg has shown ${ }^{2}$ that the intensity of reflection of

or at most in a finite number of directions. The ability to possess stable orientation in any direction in the surface of a narrow cone would require a mechanism that is difficult to imagine. It is certainly a much more artificial hypothesis than the conception of freedom for orientation in any direction.

${ }^{1}$ W. H. Bragg and W. L. Bragg, X-rays and Crystal Structure, p. 195.

${ }^{2}$ W. H. Bragg, Phil. Mag., 27, 88 I (I9I4). 
X-rays from a crystal in which the successive atomic layers are similar and similarly spaced falls off approximately according to the law

$$
E=\frac{C\left(\mathrm{I}+\cos ^{2} 2 \theta\right)}{\sin ^{2} \theta} e^{-B \sin ^{2} \theta} .
$$

In this expression $C$ and $B$ are constants, and $\theta$ is the glancing angle at which the $\mathrm{X}$-rays strike the crystal face. This law represents an average for a number of different kinds of crystals. Darwin has found, ${ }^{1}$ however, from theoretical considerations, that if all the electrons are in the midplanes of the atomic layers to which they belong, the intensity should fall off according to the law, ${ }^{2}$

$$
E_{m}=\frac{C^{\prime}\left(\mathrm{I}+\cos ^{2} 2 \theta\right)}{\sin \theta \cos \theta} e^{-B \sin ^{2} \theta} .
$$

This expression corresponds to reflection from a crystal composed of atoms of the Rutherford type which is magnetically saturated perpendicularly to the crystal face. Expression (I) indicates the reflection from an unmagnetized crystal, in which the magnetic axes of the atoms are oriented at random. The ratio of the intensity of reflection from the magnetized to that from the unmagnetized crystal should therefore be

$$
\stackrel{E_{m}}{{ }_{E}}=\frac{C^{\prime}}{C} \tan \theta
$$

For our approximate calculations, since $\theta$ is never large we may substitute $\sin \theta$ for $\tan \theta$. Thus in virtue of the relation $n \lambda=2 D \sin \theta$ this ratio becomes,

$$
\begin{aligned}
\frac{E_{m}}{E} & =\frac{C^{\prime}}{C} \cdot \frac{n \lambda}{2 D} \\
& =k n,
\end{aligned}
$$

where $k=C^{\prime} \lambda / 2 C D$ is a coefficient independent of the order of reflection $n$. By extrapolation for the intensity of the X-ray spectrum line of zero order, where theoretical considerations show that $E_{m} / E$ must be unity, it has been shown by one of the writers ${ }^{3}$ that for the first order reflection

1 C. G. Darwin, Phil. Mag., 27, 675 (rgr4).

2 This expression assumes that the effective absorption coefficient of the $\mathrm{X}$-rays in the crystal is the same for all orders of reflection. It is possible that the effective value is less in the higher orders. This would mean even less rapid diminution of intensity for the higher orders, and the predicted change due to magnetization would be even greater.

${ }^{3}$ A. H. Compton, Phys. Rev., 9, 52 (IgI7). It should be noted that Bragg's expression (I) must of necessity break down when applied to estimate the intensity of the zero order spectrum line. The extrapolation here referred to is accordingly based upon certain particular arrangements of the electrons in the atoms. Any reasonable arrangement, however, will lead to values for this ratio which do not differ greatly from those here given, so these values cannot be much in error. 
from a cleavage face of rock-salt the ratio $E_{m} / E$ is about 1.5 and for calcite is about 1.2. The ratio is doubtless of the same order of magnitude for other crystals. Taking $\mathrm{I} .3$ as a mean value when $n=\mathrm{I}$, this gives in place of equation (3),

$$
E_{m} / E=\mathrm{I} \cdot 3 n .
$$

The intensity of reflection should therefore be increased by a factor of about I.3 in the first order, 2.6 in the second order, 3.9 in the third order and 5.2 in the fourth order, if the crystal is composed wholly of atoms of the Rutherford type which are oriented with their axes parallel to the magnetic field.

It might be supposed that in the case of magnetite the iron atoms only would be oriented by the magnetic field while the oxygen atoms would remain unaffected. While this would reduce to some extent the change in intensity to be expected, it is obvious that the change would still be comparatively large. It is thus apparent that the hypothesis that atoms of the Rutherford type constitute the ultimate magnetic particles is incompatable with the negative result of our experiment.

Theoretical considerations seem to lead to the conclusion that, in order for an atom consisting of electrons revolving in orbits to be stable, the orbits must all lie in the same plane. It is therefore difficult to defend the hypothesis of a magnetic atom in which the electrons lie in different planes. It will be profitable, nevertheless, to consider the change to be expected in the intensity of the reflected X-ray beam if the atoms of the reflecting crystal have a more isotropic form.

Perhaps the most nearly isotropic form that has been suggested for the iron atom is that proposed by Hull ${ }^{1}$ to account for the X-ray spectrum obtained from an iron crystal. This atom has two electrons near the center, the remaining 24 electrons being situated at the corners of three similarly oriented, concentric cubes whose diagonals are in the ratio $\frac{1}{4}: \frac{1}{2}: \mathrm{I}$. The diagonal of the outer cube, at whose corners the "valence electrons" are situated, he estimates as $\mathrm{I} .25 \times 1 \mathrm{IO}^{-8} \mathrm{~cm}$. It is possible to calculate the order of magnitude of the effect due to the change in the orientation of such atoms by the help of the formula given by Darwin ${ }^{2}$ and one of the writers, ${ }^{3}$ in which the energy of the different orders of the reflected $\mathrm{X}$-ray beam is expressed as a function of the distances of the various electrons from the mid-planes of the layers of atoms to which they belong and the distance between the successive layers of atoms. Since the value of $D$ for the natural (III) faces of magnetite is known, 4.Io

1 A. W. Hull, Phys. Rev., 9, 85 (I9I7).

${ }^{2}$ C. G. Darwin, loc. cit.

${ }^{3} \mathrm{~A} . \mathrm{H}$. Compton, loc. cit. 
$\times \mathrm{IO}^{-8} \mathrm{~cm}$., the intensity of the reflected beam can thus be determined for any given orientation of the atoms and for any order of reflection.

In Table I. we have shown the results of this calculation for different orders of reflection from magnetite. The numbers given in the second and third columns represent the ratio of the intensity when the crystal

TABLE I.

\begin{tabular}{c|c|c}
\hline Order. & $E_{1} / E_{u}$ & $E_{2} / E_{u \cdot}$ \\
\hline $1 \ldots \ldots \ldots \ldots \ldots$ & 1.000 & 1.004 \\
$2 \ldots \ldots \ldots \ldots \ldots$ & .96 & 1.03 \\
$3 \ldots \ldots \ldots \ldots \ldots$ & .86 & 1.09 \\
$4 \ldots \ldots \ldots \ldots$ & .51 & 1.09 \\
\hline
\end{tabular}

is magnetized to its value when unmagnetized. The intensity $E_{u}$ for the unmagnetized crystal is calculated for random orientation of the atoms. In the second column the intensity $E_{1}$ is calculated for the case in which the cube face of the atom is parallel with the crystal face, i.e., on the assumption that the magnetic axis of the atom is perpendicular to its cube face. The intensity $E_{2}$, used in the third column, is estimated for the atoms with their cube diagonals perpendicular to the crystal face. In performing these calculations it has been assumed that the oxygen atoms in magnetite have their electrons arranged in the same manner as in calcite ${ }^{1}$ and that they are unaffected by the magnetic field. It is also supposed that parallel to the (III) planes of magnetite, which were those used in the experiment, all the atomic layers are similar and are similarly spaced. Whether or not these assumptions are strictly accurate, the change to be expected if the atoms are rotated by a magnetic field should be of the order of magnitude here estimated.

It is obvious from these calculations that if the iron atoms in a magnetite crystal are of the type suggested by Hull, a rotation of the atoms due to magnetization would have easily been detected in our experiments.

The surprisingly large calculated variation in the intensity of the reflected beam for a magnetic atom as nearly isotropic as that proposed by Hull makes it appear improbable that any reasonable fixed distribution of the electrons in the iron atom would be so isotropic as to make possible a rotation of the atoms without detection. There remains the possibility that the electrons may be arranged at random as a sort of atmosphere about the atomic nucleus. While this would result in an atom which would be on the average isotropic, it is obvious that such an atom would not as a whole have any polarity and could not therefore

\footnotetext{
${ }^{1}$ A. H. Compton, loc. cit.
} 
as a whole act as an elementary magnet. Subject to some uncertainty with regard to the extent of the orientation of the ultimate magnetic particles, the conclusion may therefore be drawn from our experiment that the elementary magnet is very probably not the atom as a whole.

If one admits the possibility of only a very slight change in orientation of the elementary magnets, the effect of magnetization might have escaped notice even though the atoms are not isotropic. For example, if the iron atoms are built on Bohr's model, the magnetic moment per atom will be so great that the saturation intensity of magnetization of iron would require a maximum change of orientation of only 6 degrees. This would produce an effect which if an iron crystal had been used would have just been detectable in our experiment, but with the crystal of magnetite which we employed would have been considerably smaller than the errors of measurement. However, the argument given above for the nearly complete orientation of the elementary magnets seems to eliminate the necessity of considering this possibility.

Other Possible Explanations of Ferromagnetism.-There remain four possible explanations of the magnetic properties of iron: (I) Only a small fraction of the total number of atoms may be subject to orientation by an external magnetic field, these atoms possessing a very large magnetic moment. (2) The magnetic properties of the atom may be due to a few electrons revolving in very small orbits, a change in the orientation of these orbits occurring without any change in the orientation of the remainder of the atom. (3) The positive nucleus of the atom may be magnetic and subject to orientation by the magnetic field. And (4) the electron itself may be magnetic and subject to orientation by the magnetic field.

I. A minimum limit may be placed upon the number of atoms of the Rutherford type which may be turned around by the magnetic field without being detected in our experiment. It has been shown above that a complete orientation of all the atoms should increase the intensity of reflection in the fourth order by a factor of 5 . Thus the orientation of $\mathrm{I} / 500$ th of the atoms would be sufficient to produce a change of $\mathrm{I}$ per cent. Since in our experiment we worked at only about .4 of the saturation intensity of the magnetite crystal, for complete saturation it is possible that I atom out of 200 might be turned by the magnetic field.

Considerations of symmetry make it appear improbable that in a homogeneous crystal of similarly arranged atoms so small a fraction of the atoms should be subject to orientation by a magnetic field. But perhaps a more serious difficulty with this hypothesis is the large magnitude of the magnetic moment which it is necessary to assign to the 
particular iron atoms which are subject to orientation. The magnetic moment per atom in magnetite is

$$
\frac{\text { saturation intensity of magnetization }}{\text { number of atoms per cubic } \mathrm{cm} .}=5 . \mathrm{I} \times \mathrm{IO}^{-21} \mathrm{e} . \mathrm{m} . \mathrm{u} \text {. }
$$

This value ${ }^{1}$ is of the same order of magnitude as the moment $\mathrm{I} 2 \times \mathrm{IO}^{-21}$ e.m.u. of an atom of oxygen, ${ }^{2}$ for example, which can be calculated on the basis of Langevin's theory of a paramagnetic gas. If, however, only $\mathrm{I}$ in 200 of the atoms is subject to orientation by the external field, the magnetic moment of the mobile atoms must be $200 \times 5 \times$ IO $^{-21}$ or $\mathrm{I} \times \mathrm{IO}^{-18}$ e.m.u. Suppose that all the electrons in this atom rotate in coplanar orbits, each with angular velocity $h / 2 \pi$, as assumed by Bohr. The magnetic moment per electron will then be $9.2 \times$ IO $^{-21}$ e.m.u., ${ }^{3}$ and for all 26 electrons will be $26 \times 9.2 \times 10^{-21}=.24 \times 10^{-18}$ e.m.u. This hypothesis would thus necessitate an atom with the apparently prohibitive magnetic moment of not less than 4 times that supposed by Bohr.

2. If the magnetic properties of magnetite are to be accounted for by a single electron in each atom rotating in an orbit whose plane can be altered without changing the orientation of the atom as a whole, it can be shown that the radius of this orbit must be less than $\mathrm{I} \times \mathrm{IO}^{-9}$ $\mathrm{cm}$. in order to account for the negative result of our experiment. A study of the relative intensities of the different orders of an X-ray spectrum line shows, however, ${ }^{4}$ that the outer electrons of an atom are at a distance of the order of $\mathrm{I} \times \mathrm{IO}^{-8} \mathrm{~cm}$. from the center of the atom. If the mobile electrons are rotating about the nucleus, this hypothesis would therefore mean that the electrons responsible for the atom's magnetic properties are in one of the inner rings whose radius is less than I/Io that of the atom. There are a number of effects, however, which are difficult to explain unless an atom's magnetic properties depend upon its surface electrons. Among these may be noted: ${ }^{5}$

I. The profound effect of chemical constitution on the magnetic properties of an atom,

2. The effect of temperature on magnetic properties,

3. The effect of mechanical jars in facilitating the orientation of the elementary magnets.

These phenomena make it appear improbable that the ultimate magnetic

\footnotetext{
$1 \mathrm{~S}$. Dushman, Theories of Magnetism, p. 44.

2 Ibid., p. 24.

${ }^{3}$ O. W. Richardson, Electron Theory of Matter, p. 395.

${ }^{4}$ A. H. Compton, Phys. Rev., 9, 52 (I9I7).

${ }^{5}$ Cf. K. T. Compton and E. A. Trousdale, loc. cit.
} 
particle consists of a ring of electrons revolving as near the center of the atom as the result of our experiment requires.

It is possible, however, that these small orbits may be those of the outer valence electrons revolving about positions of equilibrium. If this is the case, there must be intense forces acting on these electrons of a kind concerning which we have as yet no knowledge. Thus the central force required to hold an electron in so small an orbit with angular momentum sufficient to account for the magnetic moment of the iron atom in magnetite must not be less than that due to a positive charge of Io electronic units placed at the center of the orbit. In view of our ignorance concerning the character of the infra-atomic forces, it cannot perhaps be said that forces of this magnitude do not occur near the: surface of the atom. We have, however, no other reason to suspect the existence of such forces, and the assumption that they exist would mean a very radical departure from our usual ideas of electrodynamics. One would therefore wish to consider this explanation of our experiment: only as a last resort.

3. The considerations just brought forward as indicating that the magnetic properties of an atom depend in large measure upon its surface electrons are of equal weight as opposed to the hypothesis that the positive nucleus is the elementary magnet. Moreover the experiments of Barnett on magnetization ${ }^{1}$ by rotation and by Stewart on rotation by magnetization ${ }^{2}$ show that at least the major part of ferromagnetism is due to the motion of negative electricity. This is difficult to explain if the positive nucleus of the atom is the ultimate magnetic particle. Our experiment, however, brings forth no new evidence on this point.

4. It is clear that the result of our experiment is in accord with the hypothesis that the electron itself is the ultimate magnetic particle, unless the electron is assumed to have dimensions so great that a change in its orientation will produce an appreciable effect. Let us suppose with Parson $^{3}$ that the electron is a ring of electricity with a magnetic axis perpendicular to its plane, and in addition that on magnetization the axis of the electron is brought perpendicular to the reflecting surface of the crystal. If all the electrons are oriented by magnetization and if it is assumed that the different elements of the electron move under the action of the incident wave as if they were independent charged particles of definite mass, calculation then shows that the effect should not be noticeable in our experiment if the radius of the electron is less than $4 \times 10^{-10} \mathrm{~cm}$. Thus if the radius is $2 \times 10^{-10} \mathrm{~cm}$. as one of us has esti-

1 S. J. Barnett, loc. cit.

${ }^{2}$ J. Q. Stewart, loc. cit.

${ }^{3}$ A. L. Parson, Smithsonian Misc. Collections, Nov., I9I5. 
mated on the basis of the scattering of $\mathrm{X}$-rays and gamma rays, ${ }^{1}$ the hypothesis that the electron is the ultimate magnetic particle is in accord with our experimental results.

Conclusions.-The fact that on magnetizing the crystal of magnetite - the intensity of the reflected X-ray beam did not change by as much as I per cent. in any of the first four orders therefore supports the conclusion of K. T. Compton and E. A. Trousdale that the elementary magnet in a ferromagnetic substance is not a group of atoms.

Subject to the validity of our argument for the nearly complete alignment of the elementary magnets with the external magnetic field, it is also apparently impossible to explain this result on the hypothesis that the atom as a whole acts as the ultimate magnet, since an orientation of the atoms by the applied magnetic field would have made a noticeable change in the intensity of the reflected X-ray beam.

The hypotheses that only a small fraction of the atoms are turned by the magnetic field or that the magnetic effects are due to certain electrons whose orbits may change without affecting the remainder of the atom, have not appeared to be plausible explanations of our result.

Our experiment is in accord with the suggestion that the magnetic properties of matter are due either to the nucleus of the atom or to the individual electrons. Auxiliary evidence, however, indicates that the electron is the more probable elementary magnet.

This experiment was performed in the Physics Laboratory of the University of Minnesota during the winter of I9I6-I7. We take pleasure in thanking Professor K. T. Compton, of Princeton University, for his helpful suggestions in interpreting the results of this work.

\section{A. H. C.,}

Cavendish Laboratory, Cambridge.

$$
\text { O. R., }
$$

May 22, 1920.

$$
\text { Bureau of Standards, Washington. }
$$

${ }^{1}$ A. H. Compton, Phys. Rev., I4, 3I (I9I9). 